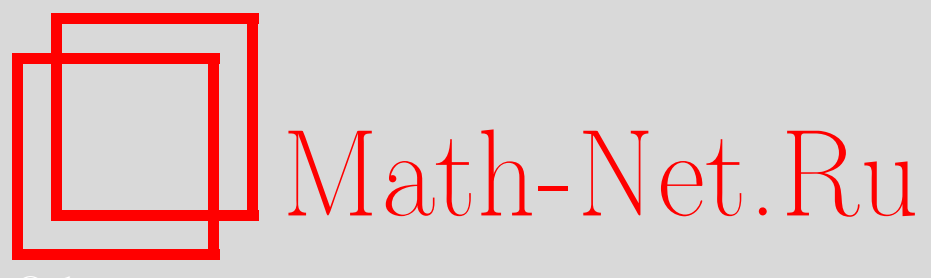

С. Ф. Лукомский, Г. С. Бердников, Ю. С. Крусс, Об ортогональности системы сдвигов масштабирующей функции на группах Виленкина, Матем. заметки, 2015, том 98, выпуск 2, 310-313

DOI: https://doi.org/10.4213/mzm10664

Использование Общероссийского математического портала Math-Net.Ru подразумевает, что вы прочитали и согласны с пользовательским соглашением http://www . mathnet.ru/rus/agreement

Параметры загрузки:

IP : 3.89 .197 .203

26 апреля 2023 г., $17: 48: 44$

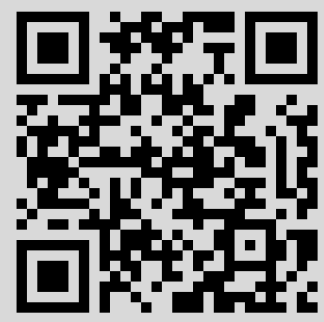




\section{Об ортогональности системы сдвигов масштабирующей функции на группах Виленкина}

\section{С. Ф. Лукомский, Г. С. Бердников, Ю. С. Крусс}

Введение. Пусть $(G, \dot{+})$ - локально компактная группа Виленкина, элементами которой являются бесконечные в обе стороны последовательности

$$
x=\left(\ldots, 0_{n-1}, x_{n}, x_{n+1}, \ldots\right), \quad x_{j}=0, \ldots, p-1,
$$

где $p$ - любое простое число; $g_{n}=\left(\ldots, 0_{n-1}, 1_{n}, 0_{n+1}, \ldots\right)$ - базисные элементы в $G$. Операция сложения + определяется как покоординатное сложение по модулю $p$, т.е.

$$
x+y=\left(x_{j} \dot{+} y_{j}\right)=\left(x_{j}+y_{j} \bmod p\right) .
$$

Пусть

$$
G_{n}=\left\{x \in G: x=\left(\ldots, 0_{n-1}, x_{n}, x_{n+1}, \ldots\right)\right\}, \quad n \in \mathbb{Z},
$$

- основная цепочка подгрупп, $G_{n}^{\perp}$ - аннуляторы подгрупп $G_{n}, X$ - группа всех характеров, $r_{n} \in G_{n+1}^{\perp} \backslash G_{n}^{\perp}-$ функции Радемахера на группе $G$. Оператор растяжения $\mathscr{A}$ в группе $G$ определяется равенством $\mathscr{A} x:=\sum_{n=-\infty}^{+\infty} a_{n} g_{n-1}$, где $x=\sum_{n=-\infty}^{+\infty} a_{n} g_{n} \in G$, в группе характеров - равенством $(\chi \mathscr{A}, x)=(\chi, \mathscr{A} x)$. Определим множества

$$
\begin{aligned}
H_{0}^{(s)} & =\left\{h \in G: h=a_{-1} g_{-1} \dot{+} a_{-2} g_{-2} \dot{+} \cdots+a_{-s} g_{-s}\right\}, \quad s \in \mathbb{N}, \\
H_{0} & =\left\{h \in G: h=a_{-1} g_{-1} \dot{+} a_{-2} g_{-2} \dot{+} \cdots+a_{-s} g_{-s}, s \in \mathbb{N}\right\} .
\end{aligned}
$$

Множество $H_{0}$ есть множество сдвигов в $G$. Оно есть аналог множества целых неотрицательных чисел.

Протасов, Фарков в работах [1]-[3] охарактеризовали все двоичные финитные всплески на $\mathbb{R}_{+}$и указали алгоритм их построения. Фарков в работах [4], [5], рассматривая масштабирующие функции $\varphi(x)$ с компактным носителем $G_{-N}$, получил необходимые и достаточные условия на маску $m_{0}(\chi)$, которая порождает ортогональный КМА. Эти условия были даны при дополнительном предположении

$$
\sum_{\alpha_{0}=1}^{p-1}\left|m_{0}\left(G_{-N}^{\perp} r_{-N}^{\alpha_{-N}} r_{-N+1}^{\alpha_{-N+1}} \ldots r_{-1}^{\alpha_{-1}} r_{0}^{\alpha_{0}}\right)\right|^{2}=1
$$

которое является необходимым для ортогональности системы сдвигов соответствующей масштабирующей функции $\varphi$. Фарков в [4]-[5], доказал, что в этом случае масштабирующая функция $\varphi$ порождает ортогональный КМА тогда и только тогда, когда маска $m_{0}$ не имеет блокированных множеств. Задача нахождения блокированных множеств является переборной и требует перебора примерно $2^{p^{N}}$ различных вариантов, что реально только при достаточно малых $p$ и $N$.

Поэтому задача построения ортогональной масштабирующей функции, не требующая перебора, является актуальной. В работах [6], [7] для построения масштабирующей функции $\varphi$ были использованы деревья, но предложенный там метод работает в случае, когда функция $|\widehat{\varphi}(\chi)|$ постоянна на смежных классах по подгруппе $G_{-1}^{\perp}$ и принимает только 2 значения: 0 или 1. В [8] удалось избавиться от условия $\operatorname{supp} \varphi(x) \subset G_{-1}$, для этого было введено понятие $N$-валидного дерева и было доказано, что ступенчатая функция $\varphi(x)$ с носителем $\operatorname{supp} \varphi(x) \subset G_{-N}$ и условием $|\widehat{\varphi}(\chi)|=0$ или 1 порождает KMА тогда и только тогда,

Работа выполнена при поддержке Российского фонда фундаментальных исследований (грант № 13-01-00102-a).

DOI: $10.4213 / \mathrm{mzm} 10664$

(C) С.Ф. Лукомский, Г. С. Бердников, Ю. С. Крусс, 
когда $\varphi(x)$ порождена $N$-валидным деревом. В настоящей заметке мы избавляемся от условия $|\widehat{\varphi}(\chi)|=0$ или 1 и указываем алгоритм построения ортогональной масштабирующей функции с единственным ограничением: $\widehat{\varphi}(\chi)-$ ступенчатая функция. Полученный алгоритм не требует перебора.

Задачу построения такой функции мы сводим к нахождению некоторого графа, который, в свою очередь, строится по произвольному $N$-валидному дереву.

1. Масштабирующая функция и КМА. Мы будем рассматривать случай, когда масштабирующая функция $\varphi$, порождающая ортогональный КMА, является ступенчатой. Множество ступенчатых функций, постоянных на смежных классах по подгруппе $G_{M}$ с носителем $\operatorname{supp}(\varphi) \subset G_{-N}$, обозначим через $\mathfrak{D}_{M}\left(G_{-N}\right), M, N \in \mathbb{N}$. Аналогично, $\mathfrak{D}_{-N}\left(G_{M}^{\perp}\right)$ есть множество ступенчатых функций, постоянных на смежных классах по подгруппе $G_{-N}^{\perp}$ с носителем $\operatorname{supp}(\varphi) \subset G_{M}^{\perp}$. Если функция $\varphi \in \mathfrak{D}_{M}\left(G_{-N}\right)$ порождает ортогональный КМА, то она удовлетворяет масштабирующему уравнению $\varphi(x)=\sum_{h \in H_{0}^{(N+1)}} \beta_{h} \varphi(\mathscr{A} x \dot{-} h)[9]$, которое можно записать в частотном виде

$$
\widehat{\varphi}(\chi)=m_{0}(\chi) \widehat{\varphi}\left(\chi \mathscr{A}^{-1}\right)
$$

где

$$
m_{0}(\chi)=\frac{1}{p} \sum_{h \in H_{0}^{(N+1)}} \beta_{h} \overline{\left(\chi \mathscr{A}^{-1}, h\right)}
$$

- маска уравнения (1). В [9] были доказаны следующие утверждения.

1) Если $\widehat{\varphi}(\chi) \in \mathfrak{D}_{-N}\left(G_{M}^{\perp}\right)$ есть решение масштабирующего уравнения (1) и система сдвигов $(\varphi(x \dot{-} h))_{h \in H_{0}}$ есть ортонормированная система, то $\varphi$ порождает ортогональный КМА.

2) Если $\widehat{\varphi}(\chi) \in \mathfrak{D}_{-N}\left(G_{M}^{\perp}\right)$, то система сдвигов $(\varphi(x \dot{-} h))_{h \in H_{0}}$ будет ортонормированной системой тогда и только тогда, когда для любых $\alpha_{-N}, \alpha_{-N+1}, \ldots, \alpha_{-1}=(0, \ldots, p-1)$

$$
\sum_{\alpha_{0}, \alpha_{1}, \ldots, \alpha_{M-1}=0}^{p-1}\left|\widehat{\varphi}\left(G_{-N}^{\perp} r_{-N}^{\alpha_{-N}} \ldots r_{0}^{\alpha_{0}} \ldots r_{M-1}^{\alpha_{M-1}}\right)\right|^{2}=1 .
$$

Таким образом, для построения ортогонального КМА нужно построить функцию $\widehat{\varphi}(\chi) \in$ $\mathfrak{D}_{-N}\left(G_{M}^{\perp}\right)$, которая является решением масштабирующего уравнения (1) и для которой выполнены условия (3). Именно одновременное выполнение этих двух условий представляет главную сложность. Для достижения этих двух целей и появились блокированные множества. Мы предлагаем решение этой задачи без использования блокированных множеств.

\section{2. Построение ортогональной масштабирующей функции.}

ОПредЕлЕНиЕ 2.1. Пусть $N$ - натуральное число, $p$ - простое. Под $N$-валиднъим деревом мы будем понимать дерево, ориентированное от листа к корню и удовлетворяющее следующим условиям:

1) корень и все вершины вплоть до $(N-1)$-го уровня имеют значение равное нулю;

$2)$ любой путь $\left(\alpha_{k} \rightarrow \alpha_{k+1} \rightarrow \cdots \rightarrow \alpha_{k+N-1}\right)$ длины $N-1$ присутствует в дереве ровно один раз; здесь $\alpha_{i}=0, \ldots, p-1$.

Выберем $N$-валидное дерево $T$ и будем строить по нему масштабирующую функцию.

1) По дереву $T$ строим новое дерево $\widetilde{T}$ следующим образом. Заменяем последовательность из $N$ нулей, заканчивающуюся корнем дерева, на одну вершину со значением $\left(0_{N}\right.$, $\left.0_{N-1}, \ldots, 0_{1}\right)$. Все вершины $(N+1)$-го уровня дерева $T$ теперь связаны с этой вершиной в дереве $\widetilde{T}$. Она является корнем дерева $\widetilde{T}$. Без изменения связей переобозначаем остальные вершины. Если в дереве $T$ с вершины $\alpha_{N}$ начинался путь из $N$ элементов в направлении к корню

$$
\alpha_{N} \rightarrow \alpha_{N-1} \rightarrow \cdots \rightarrow \alpha_{1}
$$


то в новом дереве $\widetilde{T}$ данная вершина будет иметь значение, равное $N$-мерному вектору $\left(\alpha_{N}, \alpha_{N-1}, \ldots, \alpha_{1}\right)$. В силу условия $N$-валидности дерева $T$ каждому такому вектору в дереве $\widetilde{T}$ соответствует единственная вершина. Также, если мы обозначим $\operatorname{height}(T)=H$, $\operatorname{height}(\widetilde{T})=\widetilde{H}$, то очевидно $\widetilde{H}=H-N+1$.

2) Теперь по дереву $\widetilde{T}$ строим граф Г. Каждую вершину $\bar{\alpha}_{N}=\left(\alpha_{N}, \alpha_{N-1}, \ldots, \alpha_{1}\right)$ дерева $\widetilde{T}$ свяжем со всеми вершинами низшего уровня, имеющими вид $\left(\alpha_{N-1}, \ldots, \alpha_{1}, \alpha_{0}\right)$, т.е. первые $N-1$ элементов этой вершины совпадают с последними $N-1$ элементами вершины $\bar{\alpha}_{N}$. Вершины, с которыми вершина $\bar{\alpha}_{N}$ связана, мы будем обозначать $\left(\alpha_{N-1}, \ldots, \alpha_{1}\right.$, $\left.\widetilde{\alpha}_{0}\right)$. То есть $\alpha_{0} \in\left\{\widetilde{\alpha}_{0}\right\}$ тогда и только тогда, когда вершина $\bar{\alpha}_{N}$ связана с вершиной $\left(\alpha_{N-1}\right.$, $\left.\ldots, \alpha_{1}, \alpha_{0}\right)$ в графе $\Gamma$.

3) Обозначим

$$
\lambda_{\alpha_{-N}, \alpha_{-N+1}, \ldots, \alpha_{-1}, \alpha_{0}}=\left|m_{0}\left(G_{-N}^{\perp} r_{-N}^{\alpha_{-N}} r_{-N+1}^{\alpha_{-N+1}} \ldots r_{-1}^{\alpha_{-1}} r_{0}^{\alpha_{0}}\right)\right|^{2} .
$$

Если вершина $\left(\alpha_{-N}, \alpha_{-N+1}, \ldots, \alpha_{-1}\right)$ в графе $\Gamma$ связана с вершинами

$$
\left(\alpha_{-N+1}, \alpha_{-N+2}, \ldots, \alpha_{-1}, \widetilde{\alpha}_{0}\right)
$$

то значения маски определяем так, чтобы

$$
\sum_{\widetilde{\alpha}_{0}} \lambda_{\alpha_{-N}, \alpha_{-N+1}, \ldots, \alpha_{-1}, \widetilde{\alpha}_{0}}=1, \quad \lambda_{\alpha_{-N}, \alpha_{-N+1}, \ldots, \alpha_{-1}, \alpha_{0}}=0 \quad \text { для всех } \quad \alpha_{0} \notin\left\{\widetilde{\alpha}_{0}\right\} .
$$

Также определим $m_{0}\left(G_{-N}^{\perp}\right)=1$, откуда следует, что $\lambda_{0,0, \ldots, 0}=1$.

Чтобы сформулировать основной результат, введем дополнительные обозначения. Сначала заметим, что условие (3) ортонормированности системы сдвигов функции $\varphi(x)$, можно переписать в следующем виде: для любых $\alpha_{-N}, \alpha_{-N+1}, \ldots, \alpha_{-1}=0, \ldots, p-1$,

$$
\begin{aligned}
1 & =\sum_{\alpha_{0}, \alpha_{1}, \ldots, \alpha_{M-1}=0}^{p-1}\left|\widehat{\varphi}\left(G_{-N}^{\perp} r_{-N}^{\alpha_{-N}} \ldots r_{-1}^{\alpha_{-1}} r_{0}^{\alpha_{0}} \ldots r_{M-1}^{\alpha_{M-1}}\right)\right|^{2} \\
& =\sum_{\alpha_{0}=0}^{p-1} \lambda_{\alpha_{-N}, \alpha_{-N+1}, \ldots, \alpha_{0}} \sum_{\alpha_{1}=0}^{p-1} \lambda_{\alpha_{-N+1}, \alpha_{-N+2}, \ldots, \alpha_{1}} \ldots \sum_{\alpha_{M-2}=0}^{p-1} \lambda_{\alpha_{M-N-2}, \alpha_{M-N-1}, \ldots, \alpha_{M-2}} \\
& =\sum_{\alpha_{M-1}=0}^{p-1} \lambda_{\alpha_{M-N-1}, \alpha_{M-N}, \ldots, \alpha_{M-1}} \lambda_{\alpha_{M-N}, \alpha_{M-N+1}, \ldots, \alpha_{M-1}, 0} \ldots \lambda_{\alpha_{M-1}, 0, \ldots, 0} .
\end{aligned}
$$

Теперь зададим последовательность $N$-мерных массивов

$$
A^{(n)}=\left(a_{i_{1}, i_{2}, \ldots, i_{N}}^{(n)}\right)_{i_{1}, i_{2}, \ldots, i_{N}=0}^{p-1}
$$

рекуррентно через их элементы:

$$
\begin{aligned}
& a_{i_{1}, i_{2}, \ldots, i_{N}}^{(0)}=\lambda_{i_{1}, i_{2}, \ldots, i_{N}, 0} \lambda_{i_{2}, i_{3}, \ldots, i_{N}, 0,0} \ldots \lambda_{i_{N}, 0, \ldots, 0}, \\
& a_{i_{1}, i_{2}, \ldots, i_{N}}^{(n)}=\sum_{j=0}^{p-1} \lambda_{i_{1}, i_{2}, \ldots, i_{N}, j} a_{i_{2}, i_{3}, \ldots, i_{N}, j}^{(n-1)} .
\end{aligned}
$$

Мы будем говорить, что элемент массива $a_{i_{1}, i_{2}, \ldots, i_{N}}^{(s)}$ соответствует вершине $\left(i_{1}, i_{2}, \ldots, i_{N}\right)$. Пользуясь новыми обозначениями, условие ортонормированности (5) можно переформулировать в следующем виде.

Система сдвигов по функции $\varphi(x) \in \mathfrak{D}_{M}\left(G_{-N}\right)$ будет ортонормированной тогда и только тогда, когда для любых $i_{1}, i_{2}, \ldots, i_{N}$ имеем $a_{i_{1}, i_{2}, \ldots, i_{N}}^{(M)}=1$; иными словами, когда массив $A^{(M)}$ состоит только из единиц. 
Лемма 1. В массиве $A^{(0)}$ на местах, соответствующих вершинам уровня $s \leqslant N$ в дереве $\widetilde{T}$, стоят единиць.

Лемма 2. Пусть по $N$-валидному дереву $T$ построены дерево $\widetilde{T}$, граф $\Gamma$ и определены значения маски $m_{0}(\chi)$ так, как указано в равенствах (4). Пусть $\left(A^{(n)}\right)_{n=0}^{\infty}$ - последовательность массивов, определяемая равенствами (6) и (7). Тогда в массиве $A^{(n)}$ элементы, соответствующие вериинам уровня $s \leqslant N+n$ в дереве $\widetilde{T}$, равны единице.

Из этих лемм сразу следует

Теорема. Пусть по $N$-валидному дереву $T$ построены дерево $\widetilde{T}$, граф $\Gamma$ и определенъ значения маски $m_{0}(\chi)$ так, как указано в равенствах (4). Пусть $\widetilde{H}=\operatorname{height}(\widetilde{T})$. Тогда равенство

$$
\widehat{\varphi}(\chi)=\prod_{k=0}^{\infty} m_{0}\left(\chi \mathscr{A}^{-k}\right) \in \mathfrak{D}_{-N}\left(G_{M}^{\perp}\right)
$$

определяет ортогональную масштабирующую функцию $\varphi(x) \in \mathfrak{D}_{M}\left(G_{-N}\right)$, причем $M \leqslant$ $\widetilde{H}-N$.

ЗАмечание. Обозначим через $\Phi_{N}$ совокупность функций $f_{N}:\{0,1, \ldots, p-1\}^{N} \rightarrow[0,1]$ и выберем функцию $\Lambda \in \Phi_{N+1}$. Функцию $\Lambda$ можно рассматривать как $(N+1)$-мерный массив $\Lambda=\left(\lambda_{i_{1}, i_{2}, \ldots, i_{N}, i_{N+1}}\right)$. Тогда равенства (7) определяют дискретную динамическую систему $\Lambda: \Phi_{N} \rightarrow \Phi_{N}$, а равенство (6) задает начальную точку траектории. Теорема указывает класс дискретных систем $\Lambda$, у которых траектория, выходящая из начальной точки (6), имеет неподвижную точку.

В заключение отметим роль авторов в подготовке настоящей статьи. Теорему при $N=$ $1, p=3$ получил Лукомский, при $N=1$ и произвольном $p-$ Крусс, при любых $N$ и $p-$ Бердников.

\section{СПИСОК ЦИТИРОВАННОЙ ЛИТЕРАТУРЫ}

[1] В. Протасов, Ю. Фарков, Матем. сб., 197:10 (2006), 129-160. [2] Ю. Фарков, УМН, 62:6 (2007), 189-190. [3] В. Протасов, Матем. сб., 198:11 (2007), 135-152. [4] Ю. Фарков, Изв. РАН. Сер. матем., 69:3 (2005), 193-220. [5] Ю. Фарков, Матем. заметки, 82:6 (2007), 934-952. [6] S. Lukomskii, Trees in Wavelet Analysis on Vilenkin Groups, arXiv: 1303.5635. [7] С. Лукомский, Докл. РАН, 457:1 (2014), 24-27. [8] S. Lukomskii, G. Berdnikov, N-Valid Trees in Wavelet Theory on Vilenkin Groups, arXiv: 1412.3096. [9] S. Lukomskii, J. Fourier Anal. Appl., 20:1 (2014), 42-65.

\section{С. Ф. Лукомский}

Поступило

Саратовский государственный

университет им. Н. Г. Чернышевского

E-mail: LukomskiiSF@info.sgu.ru

\section{Г. С. Бердников}

Саратовский государственный университет им. Н. Г. Чернышевского E-mail: evrointelligent@gmail.com

\section{Ю. C. Крусс}

Саратовский государственный университет им. Н. Г. Чернышевского E-mail: KrussUS@gmail.com 